\title{
A certain subclass of analytic functions associated with the quasi-subordination
}

\author{
Parviz Arjomandinia and Hossein Rahimpoor
}

\begin{abstract}
In the present paper we study quasi-subordination under a multivalent function and we consider a certain subclass of (normalized) analytic functions based on quasi-subordination. Applications and consequences of the main results are also cosidered which some of them extend the earlier issues.
\end{abstract}

Mathematics Subject Classification (2010): 30C45, 30C80.

Keywords: Subordination, quasi-subordination, starlike functions, conex functions, univalent functions.

\section{Introduction}

Let $\mathcal{A}$ be the class of (normalized) analytic functions $f(z)$ in the open unit disk

$$
\mathbb{D}=\{z \in \mathbb{C}:|z|<1\},
$$

which have Taylor series expansion

$$
f(z)=z+a_{2} z^{2}+\ldots ; \quad(z \in \mathbb{D}) .
$$

We denote by $S$ the subclass of $\mathcal{A}$ containing univalent functions. For two analytic functions $f, g$ we say that $f$ is subordinate to $g$ (or $g$ is superordinate to $f$ ), and write $f \prec g($ or $f(z) \prec g(z))$ if there exists an analytic function $w(z)$,

$$
w(z) \in \Omega=\{w:|w(z)| \leqslant|z|, z \in \mathbb{D}\}
$$

such that $f(z)=g(w(z))$. In the special case if $g$ is univalent in $\mathbb{D}$, then we have the following equivalence

$$
f(z) \prec g(z) \Longleftrightarrow f(0)=g(0) \text { and } f(\mathbb{D}) \subseteq g(\mathbb{D}) .
$$

A survey on articles shows that the notation of subordination was used frequently in the literature, see for example $[4,5,6]$. As an example, consider the following two 
classes of (normalized) analytic functions,

$$
S^{*}(\phi)=\left\{f \in \mathcal{A}: \frac{z f^{\prime}(z)}{f(z)} \prec \phi(z), z \in \mathbb{D}\right\}
$$

and

$$
K(\phi)=\left\{f \in \mathcal{A}: 1+\frac{z f^{\prime \prime}(z)}{f^{\prime}(z)} \prec \phi(z), z \in \mathbb{D}\right\},
$$

where $\phi(z)$ is analytic in $\mathbb{D}$ with $\phi(0)=1$. For $\phi(z)=\frac{1+z}{1-z}$ we obtain the well-known classes $S^{*}$ and $K$ of starlike and convex functions, respectively. By taking

$$
\phi(z)=\frac{1+(1-2 \alpha) z}{1-z}, 0 \leq \alpha<1
$$

in (1.1) and (1.2) we obtain the class of starlike and convex functions of order $\alpha$, respectively, while the choice

$$
\phi(z)=\left(\frac{1+z}{1-z}\right)^{\alpha}
$$

with $0<\alpha \leq 1$ gives the class of strongly starlike and strongly conex functions of order $\alpha$, respectively.

As an extension of subordination, Robertson [7] (see also [1]) introduced the concept of quasi-subordination. Let $f, g$ be analytic functions. We say that $f$ is quasisubordinated to $g$, and write $f \prec_{q} g$ if there exist analytic functions $\phi$ and $w$ with $|\phi(z)| \leq 1$ and $w(z) \in \Omega$ such that $f(z)=\phi(z) g(w(z))$. It is clear that for $\phi(z)=1$ we have $f \prec g$. In [3] authors considered the following two classes:

$$
S^{*}(n, A, B)=\left\{f \in \mathcal{A}: \frac{z f^{\prime}(z)}{f(z)} \prec \frac{1+A z^{n}}{1+B z^{n}}, z \in \mathbb{D}\right\}
$$

and

$$
K(n, A, B)=\left\{f \in \mathcal{A}: 1+\frac{z f^{\prime \prime}(z)}{f^{\prime}(z)} \prec \frac{1+A z^{n}}{1+B z^{n}}, z \in \mathbb{D}\right\}
$$

with $-1 \leq B<A \leq 1$, and proved certain results about the subordination properties of these two classes. Applying the notation of quasi-subordination we define the following two classes.

Definition 1.1. Let $n \in \mathbb{N}, \lambda \in \mathbb{C}-\{0\}$ and $-1 \leq B<A \leq 1$. We say that $f \in \mathcal{A}$ is in the class $S_{q}^{*}(n, \lambda, A, B)$ if there exists a $-\frac{\pi}{2}<\theta<\frac{\pi}{2}$, such that

$$
e^{i \theta}\left(1+\frac{1}{\lambda}\left(\frac{z f^{\prime}(z)}{f(z)}-1\right)\right) \prec_{q} \frac{1}{n} \sum_{k=1}^{n} \frac{1+A z^{k}}{1+B z^{k}}
$$

Definition 1.2. Let $n \in \mathbb{N}, \lambda \in \mathbb{C}-\{0\}$ and $-1 \leq B<A \leq 1$. We say that $f \in \mathcal{A}$ is in the class $K_{q}(n, \lambda, A, B)$ if there exists a $\theta,-\frac{\pi}{2}<\theta<\frac{\pi}{2}$, such that

$$
e^{i \theta}\left(1+\frac{1}{\lambda} \frac{z f^{\prime \prime}(z)}{f^{\prime}(z)}\right) \prec_{q} \frac{1}{n} \sum_{k=1}^{n} \frac{1+A z^{k}}{1+B z^{k}}
$$


It is clear that

$$
f \in K_{q}(n, \lambda, A, B) \Longleftrightarrow z f^{\prime}(z) \in S_{q}^{*}(n, \lambda, A, B) .
$$

Note that the function $\psi(z)=\frac{1+A z^{n}}{1+B z^{n}}, A \neq B, n \in \mathbb{N}$ is multivalent and maps $\mathbb{D}$ onto a disk or a half plane.

The classes $S_{q}^{*}(n, \lambda, A, B)$ and $K_{q}(n, \lambda, A, B)$ reduce to the classes which were introduced by Janowski [2] if we consider $\theta=0, n=\lambda=1=\phi(z)$. Also, by taking $n=\lambda=1, A=1, B=-1$ and $\phi(z)=1$ the class $S_{q}^{*}(n, \lambda, A, B)$ becomes the wellknown class $S_{\theta}$ of $\theta-$ spirallike functions, (see [[6],p. 9]).

In the present paper, we aim to prove special results associated with the quasisubordination for subclasses of (normalized) analytic functions. Some consequencees and applications are also considered.

In order to prove our main results, we shall use each of the following theorems.

Theorem 1.3. ([[6],p.70]) Let $h$ be conex in $\mathbb{D}$ and let $P: \mathbb{D} \longrightarrow \mathbb{C}$ with $\operatorname{Re} P(z)>0$. If $p(z)$ is analytic in $\mathbb{D}$, then

$$
p(z)+P(z) z p^{\prime}(z) \prec h(z) \Longrightarrow p(z) \prec h(z) .
$$

Theorem 1.4. ([[6],p.86]) Let $\beta, \gamma \in \mathbb{C}$ with $\beta \neq 0$ and $n \in \mathbb{N}$. Suppose that $R_{\beta a+\gamma, n}(z)$ is the "open door function" with $\operatorname{Re}(\beta a+\gamma)>0$, (see [[6],p. 46]), and that $h(z)$ is analytic in $\mathbb{D}$ with $h(0)=a$. If

$$
\beta h(z)+\gamma \prec R_{\beta a+\gamma, n}(z)
$$

then the solution $q(z)$ of

$$
q(z)+\frac{n z q^{\prime}(z)}{\beta q(z)+\gamma}=h(z)
$$

with $q(0)=a$ is analytic in $\mathbb{D}$ and satisfies $\operatorname{Re}(\beta q(z)+\gamma)>0$. If $a \neq 0$, then the solution $q$ is given by

$$
q(z)=\left[\frac{\beta}{n} \int_{0}^{1}\left(\frac{H(t z)}{H(z)}\right)^{\frac{\beta a}{n}} \cdot t^{\left(\frac{\gamma}{n}\right)-1} d t\right]^{-1}-\frac{\gamma}{\beta}
$$

where

$$
H(z)=z \exp \left(\int_{0}^{z} \frac{h(t)-a}{a t} d t\right)
$$

\section{The classes $S_{q}^{*}(n, \lambda, A, B)$ and $K_{q}(n, \lambda, A, B)$}

We begin this section with the following theorem, which gives a characterization of the functions in $S_{q}^{*}(n, \lambda, A, B)$.

Theorem 2.1. Let the function $f(z)$ belongs to the class $S_{q}^{*}(n, \lambda, A, B)$. Then there exists an analytic function $p(z)$,

$$
p(z) \prec_{q} \frac{1}{n} \sum_{k=1}^{n} \frac{1+A z^{k}}{1+B z^{k}} ;
$$


such that

$$
f(z)=z \exp \left(\lambda \int_{0}^{z} \frac{e^{-i \theta} p(t)-1}{t} d t\right) .
$$

If, in addition, the analytic function $p(z)$ satisfies (2.1), then the function of the form (2.2) belongs to $S_{q}^{*}(n, \lambda, A, B)$.

Proof. Suppose that $f \in S_{q}^{*}(n, \lambda, A, B)$. For a fixed $\theta,-\frac{\pi}{2}<\theta<\frac{\pi}{2}$ the analytic function $p$ defined by

$$
p(z)=e^{i \theta}\left(1+\frac{1}{\lambda}\left(\frac{z f^{\prime}(z)}{f(z)}-1\right)\right)
$$

satisfies (2.1). An integration in (2.3) shows that

$$
f(z)=z \exp \left(\lambda \int_{0}^{z} \frac{e^{-i \theta} p(t)-1}{t} d t\right) .
$$

Conversely, let $f$ is given by (2.2), where $p(z)$ satisfies (2.1). By differentiating logarithmically of (2.4), we obtain:

$$
p(z)=e^{i \theta}\left(1+\frac{1}{\lambda}\left(\frac{z f^{\prime}}{f}-1\right)\right),
$$

so,

$$
e^{i \theta}\left(1+\frac{1}{\lambda}\left(\frac{z f^{\prime}(z)}{f(z)}-1\right)\right) \prec_{q} \frac{1}{n} \sum_{k=1}^{n} \frac{1+A z^{k}}{1+B z^{k}}
$$

and $f \in S_{q}^{*}(n, \lambda, A, B)$.

Corollary 2.2. Let the function $f(z)$ belongs to the class $K_{q}(n, \lambda, A, B)$. Then there exists an analytic function $p$,

$$
p(z) \prec_{q} \frac{1}{n} \sum_{k=1}^{n} \frac{1+A z^{k}}{1+B z^{k}},
$$

such that

$$
f(z)=\int_{0}^{z} \exp \left(\lambda \int_{0}^{w} \frac{e^{-i \theta} p(t)-1}{t} d t\right) d w, \quad(z \in \mathbb{D})
$$

Moreover, if the analytic function $p(z)$ satisfies

$$
p(z) \prec_{q} \frac{1}{n} \sum_{k=1}^{n} \frac{1+A z^{k}}{1+B z^{k}},
$$

then the function $f$ of the form (2.5) belongs to $K_{q}(n, \lambda, A, B)$.

Proof. This is a simple consequence of Theorem 2.1. In fact $f(z) \in K_{q}(n, \lambda, A, B)$ if and only if $z f^{\prime}(z) \in S_{q}^{*}(n, \lambda, A, B)$. Equivalently $g \in S_{q}^{*}(n, \lambda, A, B)$ if and only if

$$
f(z)=\int_{0}^{z} \frac{g(w)}{w} d w
$$

is in the class $K_{q}(n, \lambda, A, B)$.

Remark 2.3. Theorem 2.1 and Corollary 2.2 remain true for complex $A, B$ and $A \neq B$ too. 
Next, consider the class $S_{q}^{*}(n, \lambda, A, B)$ with complex $A, B$ satisfying $|A| \leq 1,|B| \leq 1$ and $A \neq B$.

Theorem 2.4. Let $A, B \in \mathbb{C},|A| \leq 1$ and $|B| \leq 1$ with $A \neq B$. If the function $f$ is in the class $S_{q}^{*}(n, \lambda, A, B)$, then there exist functions $f_{k}, f_{k} \in S_{q}^{*}(1, \lambda, A, B)$, $k=1,2, \cdots, n$, such that

$$
f^{n}(z)=\prod_{k=1}^{n} f_{k}(z), \quad(z \in \mathbb{D}) .
$$

On the other hand, if there exist functions $f_{k} \in S_{q}^{*}(1, \lambda, A, B)$ such that

$$
f^{n}(z)=\prod_{k=1}^{n} f_{k}(z)
$$

then $f \in S_{q}^{*}(n, \lambda, A, B)$.

Proof. Let $f \in S_{q}^{*}(n, \lambda, A, B)$. By Theorem 2.1 there exists analytic functions $p(z), \phi(z)$ and $w(z)$ with $w(z) \in \Omega$, such that

$$
p(z)=\frac{1}{n} \phi(z) \sum_{k=1}^{n} \frac{1+A w^{k}(z)}{1+B w^{k}(z)} ; \quad(z \in \mathbb{D}),
$$

and

$$
f^{n}(z)=z^{n} \exp \left(n \lambda \int_{0}^{z} \frac{e^{-i \theta} p(t)-1}{t} d t\right) .
$$

As easy calculation yields

$$
\begin{aligned}
f^{n}(z) & =z^{n} \exp \left(\lambda \int_{0}^{z} \frac{e^{-i \theta} \phi(t) \sum_{k=1}^{n}\left\{\frac{1+A w^{k}(t)}{1+B w^{k}(t)}-n\right\}}{t} d t\right) \\
& =z^{n} \exp \left(\lambda \int_{0}^{z} \sum_{k=1}^{n}\left(e^{-i \theta} \phi(t) \frac{1+A w^{k}(t)}{1+B w^{k}(t)}-1\right) \frac{d t}{t}\right) \\
& =\prod_{k=1}^{n} z \exp \left(\lambda \int_{0}^{z}\left(e^{-i \theta} \phi(t) \frac{1+A w^{k}(t)}{1+B w^{k}(t)}-1\right) \frac{d t}{t}\right) \\
& =\prod_{k=1}^{n} f_{k}(z),
\end{aligned}
$$

where

$$
f_{k}(z)=z \exp \left(\lambda \int_{0}^{z}\left(e^{-i \theta} \phi(t) \frac{1+A w^{k}(t)}{1+B w^{k}(t)}-1\right) \frac{d t}{t}\right)
$$

By taking

it follows that

$$
p_{k}(z)=\phi(z) \frac{1+A w^{k}(z)}{1+B w^{k}(z)}
$$

$$
p_{k}(z) \prec_{q} \frac{1+A z^{k}}{1+B z^{k}} \prec_{q} \frac{1+A z}{1+B z} .
$$


So, the function $f_{k}(z),(k=1,2, \ldots, n)$ are in the class $S_{q}^{*}(1, \lambda, A, B)$. On the other hand, if there exist functions $f_{k} \in S_{q}^{*}(1, \lambda, A, B)$ such that

$$
f^{n}(z)=\prod_{k=1}^{n} f_{k}(z)
$$

then by Theorem 2.1 the functions $f_{k}$ must have the form:

$$
f_{k}(z)=z \exp \left(\lambda \int_{0}^{z}\left(e^{-i \theta} \phi(t) \frac{1+A w^{k}(t)}{1+B w^{k}(t)}-1\right) \frac{d t}{t}\right) .
$$

This shows that the product $\prod_{k=1}^{n} f_{k}(z)$ is a function $f^{n}(z)$ such that $f \in S_{q}^{*}(n, \lambda, A, B)$.

Here, we obtain another representation for the functions in $S_{q}^{*}(n, \lambda, A, B)$. Suppose that

$$
p(z) \prec_{q} F(n, A, B)=\frac{1}{n} \sum_{k=1}^{n} \frac{1+A z^{k}}{1+B z^{k}}
$$

with complex parameters $A, B,|A| \leq 1,|B| \leq 1, A \neq B$ and $A \neq 0, B \neq 0$. By defination, there exist analytic functions $\phi(z), w(z),(|\phi(z)| \leq 1$ and $w(z) \in \Omega)$ such that

$$
p(z)=\frac{1}{n} \phi(z) \sum_{k=1}^{n} \frac{1+A w^{k}(z)}{1+B w^{k}(z)} .
$$

If $a_{k}^{k}=A, b_{k}^{k}=B$ and $\xi_{i k}=\sqrt[k]{-1}$ for $i=1,2, \ldots, k$ and $k=1,2, \ldots, n$, then

$$
\begin{aligned}
p(z) & =\frac{1}{n} \phi(z) \sum_{k=1}^{n} \frac{1+\left(a_{k} w(z)^{k}\right.}{1+\left(b_{k} w(z)\right)^{k}} \\
& =\frac{1}{n} \phi(z) \sum_{k=1}^{n} \frac{\left(a_{k} w(z)\right)^{k}-\left(\xi_{i k}\right)^{k}}{\left(b_{k} w(z)\right)^{k}-\left(\xi_{i k}\right)^{k}} \\
& =\frac{1}{n} \phi(z) \sum_{k=1}^{n} \frac{\left(a_{k} w(z)-\xi_{1 k}\right)\left(a_{k} w(z)-\xi_{2 k}\right) \ldots\left(a_{k} w(z)-\xi_{k k}\right)}{\left(b_{k} w(z)-\xi_{1 k}\right)\left(b_{k} w(z)-\xi_{2 k}\right) \ldots\left(b_{k} w(z)-\xi_{k k}\right)} .
\end{aligned}
$$

Therefore

$$
\begin{aligned}
p(z) & =\frac{1}{n} \phi(z) \sum_{k=1}^{n} \prod_{i=1}^{k} \frac{a_{k} w(z)-\xi_{i k}}{b_{k} w(z)-\xi_{i k}} \\
& =\sum_{k=1}^{n} \frac{1}{n} \phi(z) \prod_{i=1}^{k} \frac{1+A_{i k} w(z)}{1+B_{i k} w(z)} \\
& =\sum_{k=1}^{n} p_{k}(z)
\end{aligned}
$$


where $A_{i k}=a_{k} \xi_{i k}^{k-1}, B_{i k}=b_{k} \xi_{i k}^{k-1}$ and

$$
\begin{aligned}
p_{k}(z) & =\frac{1}{n} \phi(z) \prod_{i=1}^{k} \frac{1+A_{i k} w(z)}{1+B_{i k} w(z)} \prec_{q} \prod_{i=1}^{k} \frac{1+A_{i k} z}{1+B_{i k} z} \\
& =\prod_{i=1}^{k} F\left(1, A_{i k}, B_{i k}\right) .
\end{aligned}
$$

\section{The class $M_{q}(n, \alpha, \lambda, A, B)$}

It is interesting to consider the conditions in which $1+\frac{1}{\lambda}\left(\frac{z f^{\prime}}{f}-1\right)$ and $1+\frac{1}{\lambda} \frac{z f^{\prime \prime}}{f^{\prime}}$ are joined. In [3] authors introduced the class $M(\alpha, n, A, B)$ as following

$$
M(\alpha, n, A, B)=\left\{f \in \mathcal{A}: \alpha\left(1+\frac{z f^{\prime \prime}}{f^{\prime}}\right)+(1-\alpha) \frac{z f^{\prime}}{f} \prec \frac{1+A z^{n}}{1+B z^{n}}, z \in \mathbb{D}\right\}
$$

where $\alpha \in \mathbb{R}$ and $-1 \leq B<A \leq 1$. For the class $M(\alpha, n, A, B)$ we have

$$
M(\alpha, n, A, B) \subseteq M(\alpha, 1, A, B) \subseteq M(\alpha)
$$

where $M(\alpha)$ is the class of $\alpha$-convex functions, (see [[6],p. 10]).

In the same way, we consider the class

$$
M_{q}(n, \alpha, \lambda, A, B)=\left\{f \in \mathcal{A}: \alpha\left(1+\frac{1}{\lambda}\left(\frac{z f^{\prime}}{f}-1\right)\right)+(1-\alpha)\left(1+\frac{1}{\lambda} \frac{z f^{\prime \prime}}{f^{\prime}}\right) \prec_{q} \frac{1+A z^{n}}{1+B z^{n}}\right\}
$$

where $\alpha \in \mathbb{R}$ and $-1 \leq B<A \leq 1$. By taking $\lambda=1$ and $\phi(z)=1$ (related to the definition of quasi-subordination) in (3.1) we obtain the class $M(\alpha, n, A, B)$. We aim to obtain the same result concerning this class. The next result involves a condition for finding a solution of Briot-Bouquet differential equation.

Theorem 3.1. Let $-1<B<A \leq 1, \alpha<1$ and $\lambda \in \mathbb{C}-\{0\}$. In addition, assume that

$$
|\lambda| \frac{1+A}{1+B}+|1-\lambda| \leq \sqrt{(1-\alpha)(3-\alpha)} .
$$

If $f \in M_{q}(n, \alpha, \lambda, A, B)$, then $f$ is starlike with respect to the origin. Also,

$$
\frac{z f^{\prime}(z)}{f(z)}=\left(\frac{1}{1-\alpha} \int_{0}^{1}\left(\frac{H(t z)}{H(z)}\right)^{\frac{\lambda}{1-\alpha}} t^{\frac{\alpha-\lambda}{1-\alpha}}\right)^{-1}
$$

where

$$
H(z)=z \exp \int_{0}^{z} \frac{(\phi(t)-1)+(A \phi(t)-B) w^{n}(t)}{t\left(1+B w^{n}(t)\right)} d t
$$

in which $\phi(z)$ and $w(z)$ are analytic in $\mathbb{D}$, such that $|\phi(z)| \leq 1$ and $w(z) \in \Omega$. In the special case if $\phi(z)=1$, then the condition $f \in M_{q}(n, \alpha, \lambda, A, B)$ implies $f \in S_{q}^{*}(1, \lambda, A, B)$. 
Proof. Put

$$
q(z)=1+\frac{1}{\lambda}\left(\frac{z f^{\prime}(z)}{f(z)}-1\right)
$$

By a straightforward calculation we see that the equation

$$
\alpha\left(1+\frac{1}{\lambda}\left(\frac{z f^{\prime}}{f}-1\right)\right)+(1-\alpha)\left(1+\frac{1}{\lambda} \frac{z f^{\prime \prime}}{f^{\prime}}\right)=h(z)
$$

becomes,

$$
q(z)+\frac{z q^{\prime}(z)}{\frac{\lambda}{1-\alpha} q(z)+\frac{1-\lambda}{1-\alpha}}=h(z) .
$$

By the assumption of the theorem, there exist analytic functions $\phi(z)$ and $w(z)$ in $\mathbb{D}$, $|\phi(z)| \leq 1$ and $w(z) \in \Omega$ such that

$$
h(z)=\phi(z) \frac{1+A w^{n}(z)}{1+B w^{n}(z)} .
$$

We have to verify conditions of the Theorem 1.4. We have

$$
\beta=\frac{\lambda}{1-\alpha}, \gamma=\frac{1-\lambda}{1-\alpha}, h(0)=1=a
$$

and

$$
\operatorname{Re}(\beta a+\gamma)=\operatorname{Re}\left(\frac{1}{1-\alpha}\right)>0
$$

Next, we investigate the condition

$$
\beta h(z)+\gamma \prec R_{\beta a+\gamma, m}(z)=R_{\frac{1}{1-\alpha}, 1}(z) .
$$

We know that the set $R_{\frac{1}{1-\alpha}, 1}(\mathbb{D})$ is the complex plane with slits along the half-lines $\operatorname{Re} z=0$ and $|\operatorname{Im} z| \geq \sqrt{1+\frac{2}{1-\alpha}}=\sqrt{\frac{3-\alpha}{1-\alpha}}$ (see [[6],p. 46]). Easy calculations show that

$$
\begin{aligned}
|\beta h(z)+\gamma| & \leq \frac{|\lambda|}{1-\alpha} \cdot \frac{\left|1+A w^{n}(z)\right|}{\left|1+B w^{n}(z)\right|}+\frac{|1-\lambda|}{1-\alpha} \\
& \leq \frac{|\lambda|}{1-\alpha} \cdot \frac{1+A}{1+B}+\frac{|1-\lambda|}{1-\alpha} \\
& =\frac{1}{1-\alpha}\left(|\lambda| \frac{1+A}{1+B}+|1-\lambda|\right) .
\end{aligned}
$$

So, in order to have (3.4) it must be

$$
\frac{1}{1-\alpha}\left(|\lambda| \frac{1+A}{1+B}+|1-\lambda|\right)<\sqrt{\frac{3-\alpha}{1-\alpha}}
$$

or equivalently,

$$
|\lambda| \frac{1+A}{1+B}+|1-\lambda|<\sqrt{(1-\alpha)(3-\alpha)} .
$$


If $\alpha$ and $\lambda$ satisfy in (3.5), then we have $\beta h(z)+\gamma \prec R_{\frac{1}{1-\alpha}, 1}(z)$. So, all conditions of the Theorem 1.4 are satisfied and we obtain

$$
0<\operatorname{Re}(\beta q(z)+\gamma)=\frac{1}{1-\alpha} \operatorname{Re}\left(\frac{z f^{\prime}}{f}\right)
$$

which means that $f \in S^{*}$. Also, we obtain the following representation for $\frac{z f^{\prime}}{f}$ :

$$
\frac{z f^{\prime}}{f}=\left(\frac{1}{1-\alpha} \int_{0}^{1}\left(\frac{H(t z)}{H(z)}\right)^{\frac{\lambda}{1-\alpha}} t^{\frac{\alpha-\lambda}{1-\alpha}}\right)^{-1}
$$

where

$$
\begin{aligned}
H(z) & =z \exp \int_{0}^{z} \frac{h(t)-1}{t} d t \\
& =z \exp \int_{0}^{z} \frac{(\phi(t)-1)+(A \phi(t)-B) w^{n}(t)}{t\left(1+B w^{n}(t)\right)} d t .
\end{aligned}
$$

In the special case if $\phi(z)=1$, then $h(z)=\frac{1+A w^{n}(z)}{1+B w^{n}(z)}$ and we have

$$
q(z)+\frac{z q^{\prime}(z)}{\frac{\lambda}{1-\alpha} q(z)+\frac{1-\lambda}{1-\alpha}} \prec \frac{1+A z}{1+B z}=h_{1}(z),
$$

where $h_{1}(z)$ is convex-univalent function in $\mathbb{D}$. Now by Theorem 1.3 we conclude that

$$
q(z)=1+\frac{1}{\lambda}\left(\frac{z f^{\prime}}{f}-1\right) \prec \frac{1+A z}{1+B z},
$$

hence $f \in S_{q}^{*}(1, \lambda, A, B)$.

\section{References}

[1] Altintas, O., Owa, Sh., Majorizations and quasi-subordinations for certain analytic functions, Proc. Japan Acad. Ser. A Math. Sci., 68(1992), no. 7, 181-185.

[2] Janowski, W., Some extremal problems for certain families of analytic functions, Ann. Polon. Math., 28(1973), no. 3, 297-326.

[3] Jurasinska, R., Sokol, J., Some problems for certain family of starlike functions, Math. and Com. Mod., 55(2012), no. 11-12, 2134-2140.

[4] Kanas, S., Differential subordination related to conic sections, J. Math. Anal. Appl., 317(2006), no. 2, 650-658.

[5] Kanas, S., Subordinations for domains bounded by conic sections, Bull. Belg. Math. Soc. Simon Stevin, 15(2008), no. 4, 1-10.

[6] Miller, S.S., Mocanu, P.T., Differential subordinations, theory and applications, Marcel Dekker, Jnc., New York, 2000.

[7] Robertson, M.S., Quasi-subordination and coefficient conjectures, Bull. Amer. Math. Soc., 76(1970), no. 1, 1-9. 
Parviz Arjomandinia

"Afagh" Higher Education Institute

Urmia, Iran

e-mail: p.arjomandinia@gmail.com

Hossein Rahimpoor

Department of Mathematics, Payame Noor University

P.O. Box, 19395-3697, Tehran, Iran

e-mail: rahimpoor2000@yahoo.com 